\title{
Análisis de estudios en metales pesados en zonas agrícolas de Colombia
}

\author{
Analysis of Studies in Heavy Metals \\ in Agricultural Areas of Colombia
}

\author{
Análise de Estudos em Metais Pesados \\ em Zonas Agrícolas ee Colômbia
}

\author{
Juan D. Mahecha-Pulido ${ }^{1 *}$, Juan M. Trujillo-González ${ }^{2 *}$, Marco A. Torres-Mora ${ }^{3 *}$ \\ 1 Ingeniero agrónomo, (e)MSc, \\ 2 Ingeniero agrónomo, MSc, \\ 3 Biólogo, PhD \\ * Instituto de Ciencias Ambientales de la Orinoquia Colombiana ICAOC, Grupo de Investigación en Gestión Ambiental \\ Sostenible-GIGAS. Facultad de Ciencias Básicas e Ingeniería, Universidad de los Llanos, Campus Barcelona Villavicencio, \\ Colombia \\ Email: jtrujillo@unillanos.edu.co,juandmahechap@gmail.com,mtorres38@gmail.com
}

Recibido: 21 de diciembre de 2016

Aceptado: 18 de abril de 2017

\begin{abstract}
Resumen
El recurso suelo oferta servicios ecosistémicos fundamentales entre las que se resaltan el soporte para la producción de alimentos y su importancia en la mitigación de los efectos del cambio climático debido a la dinámica del carbono. Sin embargo, actividades antrópicas como la densificación urbana, la industrialización y principalmente la agricultura aportan elementos como metales pesados, responsables de la degradación del suelo en algunas regiones del planeta. Naturalmente los suelos en su base geoquímica contienen metales pesados, en la mayoría de las regiones éstas concentraciones no representan riesgo ambiental. En este sentido, el propósito de la presente investigación fue recopilar los estudios de metales pesados desarrollados en sistemas de producción agrícola en Colombia para establecer una línea base, que permita identificar necesidades futuras de investigación en esta temática. Entre los resultados encontrados, se identificó que los metales pesados estudiados en el país son $\mathrm{Cd}>\mathrm{Pb}>\mathrm{Hg}>\mathrm{Cr}>\mathrm{Ni}>\mathrm{Cu}=\mathrm{Zn}=\mathrm{As}>\mathrm{Mn}>\mathrm{Fe}$, destacándose el $\mathrm{Cd}$, $\mathrm{Pb}, \mathrm{Hg}$ y $\mathrm{Cr}$. Sin embargo en Colombia la producción científica en la que son relacionados los metales pesados en la producción agrícola (suelo, cultivos o insumo) es relativamente baja. Asimismo, estos trabajos se localizan en la región central del país, evidenciando que regiones como la Orinoquia considerada como la frontera y despensa agrícola del país, únicamente se reportan tres estudios que fueron publicados en los últimos años. Finalmente con esto se resalta la importancia de generar investigaciones en valores de referencia para estos elementos en los suelos colombianos, que permitan establecer programas de monitoreo y de evaluación en posibles casos de contaminación.
\end{abstract}

Palabras clave: Metales pesados, Producción agrícola, Contaminación

\begin{abstract}
The soil resource offers fundamental ecosystem services, among which stand out the support for food production and its importance in mitigating the effects of climate change due to carbon dynamics. However, anthropic activities such as urban
\end{abstract}


densification, industrialization and mainly agriculture contribute elements such as heavy metals, responsible for soil degradation in some regions of the planet. Naturally the soils in their geochemical base contain heavy metals, in the majority of the regions these concentrations do not represent environmental risk. In this sense, the purpose of the present investigation was to compile the studies of heavy metals developed in agricultural production systems in Colombia to establish a baseline, which allows identifying future research needs in this subject. Among the results found, it was identified that the heavy metals studied in the country are $\mathrm{Cd}>\mathrm{Pb}>\mathrm{Hg}>\mathrm{Cr}>\mathrm{Ni}>\mathrm{Cu}=\mathrm{Zn}=\mathrm{As}>\mathrm{Mn}>\mathrm{Fe}$, highlighting $\mathrm{Cd}$, Pb, Hg and $\mathrm{Cr}$. However in Colombia The scientific production in which heavy metals are related to agricultural production (soil, crops or inputs) is relatively low. Also, these works are located in the central region of the country, evidencing that regions such as the Orinoquia considered as the border and agricultural pantry of the country, only three studies that were published in recent years are reported. Finally, this highlights the importance of generating research on reference values for these elements in Colombian soils, which allow the establishment of monitoring and evaluation programs in possible cases of contamination

Key Words: Heavy metals, Agricultural production, Contamination.

\section{Resumo}

O recurso solo oferece serviços ecossistêmicos fundamentais entre as que se realçam o suporte para a produção de alimentos e sua importância na mitigação dos efeitos da mudança climática devido à dinâmica do carbono. No entanto, atividades antrópicas como a densificação urbana, a industrialização e principalmente a agricultura contribuem elementos como metais pesados, responsáveis pela degradação do solo em algumas regiões do planeta. Naturalmente os solos em sua base geoquímica contêm metais pesados, na maioria das regiões estas concentrações não representam risco ambiental. Neste sentido, o propósito da presente investigação foi recopilar os estudos de metais pesados desenvolvidos em sistemas de produção agrícola em Colômbia para estabelecer uma linha base, que permita identificar necessidades futuras de investigação nesta temática. Entre os resultados encontrados, identificou-se que os metais pesados estudados no país são $\mathrm{Cd}>\mathrm{Pb}>\mathrm{Hg}>\mathrm{Cr}>\mathrm{Nem}>\mathrm{Cu}=\mathrm{Zn}=\mathrm{As}>\mathrm{Mn}>\mathrm{Fé}$, se destacando o $\mathrm{Cd}, \mathrm{Pb}, \mathrm{Hg}$ e Cr. No entanto em Colômbia a produção científica na que são relacionados os metais pesados na produção agrícola (solo, cultivos ou insumo) é relativamente baixa. Assim mesmo, estes trabalhos localizam-se na região central do país, evidenciando que regiões como a Orinoquia considerada como a fronteira e despensa agrícola do país, unicamente reportam-se três estudos que foram publicados nos últimos anos. Finalmente com isto se realça a importância de gerar investigações em valores de referência para estes elementos nos solos colombianos, que permitam estabelecer programas de monitorização e de avaliação em possíveis casos de contaminação.

Palavras-Chave: Metais pesados, Produção agrícola, Poluição.

\section{Introducción}

El suelo es un recurso natural resultado de las interacciones de factores bióticos y abióticos sobre un material parental, que lo convierte en un sistema dinámico, multifuncional con la capacidad de ofertar bienes y servicios de vital importancia para el desarrollo de las sociedades (Jenny, 1994; Doran y Parkin, 1994; Karlen et al., 1997; COM, 2002; Buol et al., 2011; Bouma et al., 2012). Las funciones del suelo consideradas como aquellas capacidades que se relacionan con la agricultura, el medio ambiente, la protección de la naturaleza y la arquitectura del paisaje (Karlen et al., 1997). Garbisu et al., 2007, describe estas funciones en detalle como: producción de biomasa y alimentos, ciclado de nutrientes, regulación hídrica, interacciones ambientales (almacenamiento, filtrado y transformación), hábitat biológico, reserva genética, fuente de materias primas, patrimonio físico y cultural, y plataforma de estructuras antrópicas (edificaciones y vías). La importancia del suelo para el ciclado de los nutrientes, la regulación hídrica y como filtro de contaminantes, han conducido a que en los últimos años el suelo sea reconocido como factor clave en la producción de cultivos, en la purificación del agua y de la atmósfera (Duval et al., 2013). En definitiva estas funciones de manera singular o in- tegrada, pueden responder a los necesidades actuales de la sociedad (McBratney et al., 2014).

Algunos investigadores consideraban al suelo como un recurso con capacidad ilimitada para soportar y asimilar contaminantes, característica que se ha desestimado actualmente, debido a que las propiedades químicas del suelo están influenciadas por el ambiente y por la dinámica antrópica, lo que lo convierte en un sistema dinámico en el espacio y en el tiempo (Montanarella, 1999; Brevik et al., 2015; Gutiérrez et al., 2016).

Los metales pesados son los contaminantes que mayormente se asocian al suelo, con origen natural cuando proviene de material parental o antrópico cuando la fuente son actividades desarrolladas por el hombre (Mico et al., 2007, Sánchez, 2010). Según Kabata-Pendias (2004) y Ceccon, 2008, en los últimos 100 años las concentraciones de estos compuestos metálicos han aumentado debido a las actividades antrópicas, donde se destaca la agricultura como una fuente no puntual. Asimismo, Alloway 2013 menciona que en el aumento de las concentraciones tienen participación fuentes naturales. Todo esto representaría un riesgo de salud pública, principalmente en los países en vías de desarrollo (Nava-Ruíz, y Méndez-Armenta, 2011). 
La agricultura moderna se ha considerado como uno de las mayores fuentes de metales pesados, debido al uso de los fertilizantes, especialmente los fosforados y nitrogenados, los plaguicidas, los compost derivados de residuos y lodos de plantas depuradoras de agua y el Estiércol (Tiller, 1989; Adriano, 2001). En la tabla 1, se presentan estimaciones de metales pesados presentes en insumos típicos de los sistemas agrícolas.

En la actualidad hay un interés de los científicos en realizar investigaciones relacionadas con los metales pesados en el mundo (Yi y Sung K., 2015; TrujilloGonzález et al., 2016; Sam et al., 2016; Khaledian et al., 2016), debido a la problemática ambiental que estos representan por ser elementos tóxicos que afectan considerablemente el suelo, el aire y las fuentes hídricas y consecuentemente la salud humana (Mico, 2005; Alloway, 2013; Yacomelo, 2014).

En este sentido, el propósito de la presente investigación fue recopilar los estudios de metales pesados desarrollados en sistemas de producción agrícola en Colombia y así establecer una línea base que permita, identificar vacíos de información, reconocer los avances y proyectar nuevas investigaciones en este campo, que contribuyan en la gestión del recurso suelo.

\section{Materiales y métodos}

El análisis de la información relacionada con la importancia del recurso suelo en actividades agrícolas y la base de información sobre estudios de metales pesados en Colombia, se recopiló a través de artículos científicos e informes de investigación disponibles en las bases de datos (Science Direct y Springer) y en portales de búsqueda académica (Google scholar, researchgate). La espacialización de los estudios se realizó a través de software ArcGis versión 10.1. A partir de esta información se realizó un análisis crítico, que permitiera plantear las perspectivas y desafíos de investigaciones futuras.

\section{Resultados y discusión}

En la tabla 2, se encuentran algunos de los estudios, en los que se evalúan metales pesados en diferentes componentes de la producción agrícola en Colombia (Suelo, especies vegetales o insumos). A partir de esta revisión se identificó que los metales que tienen mayor atención se presentan en el siguiente orden $\mathrm{Cd}>\mathrm{Pb}>\mathrm{Hg}$ $>\mathrm{Cu}>\mathrm{Cr}>\mathrm{Ni}=\mathrm{Zn}>\mathrm{As}>\mathrm{Fe}>\mathrm{Mn}>\mathrm{Mg}$. En la región Andina y costa Norte de Colombia se localizan la mayoría de las investigaciones de metales pesados, mientras que para la región de la Orinoquia, considerada por el CONPES 3797 como la última frontera agrícola del país y donde en los próximos años se enfocará la producción agropecuaria (DNP, 2014), apenas se reportan cuatro estudios que analizan directamente los metales pesados en sistemas agrícolas (Jamioy-Orozco et al., 2015; Mahecha-Pulido et al., 2015; Ramírez y Navarro, 2015; Trujillo-González et al., 2017) (Figura 1).

Tabla 1. Concentración de metales pesados $(\mathrm{mg} / \mathrm{Kg})$ adicionados al suelo a través de diferente fuente.

\begin{tabular}{|c|c|c|c|c|c|}
\hline Metal & $\begin{array}{l}\text { Fertilizantes } \\
\text { fosforados }\end{array}$ & $\begin{array}{l}\text { Fertilizantes } \\
\text { nitrogenados }\end{array}$ & Fitosanitarios & Compost & Lodos \\
\hline $\mathrm{Cd}$ & $0,1-170$ & $0,05-8,5$ & $1-38-1,94$ & $0,3-0,8$ & $2-1500$ \\
\hline $\mathrm{Co}$ & $1-12$ & $5,4-12$ & $0,2-1,9$ & $0,3-24$ & $2-260$ \\
\hline $\mathrm{Cr}$ & $66-245$ & $3,2-19$ & 13 & $5,3-55$ & $20-40600$ \\
\hline $\mathrm{Cu}$ & $1-300$ & $1-15$ & $12-50$ & $2-60$ & $50-3300$ \\
\hline $\mathrm{Mn}$ & $40-2000$ & - & $15-205$ & $30-550$ & $60-3900$ \\
\hline $\mathrm{Ni}$ & $7-38$ & $7-34$ & $0,8-14$ & $7,8-30$ & $16-5300$ \\
\hline $\mathrm{Pb}$ & $7-225$ & $2-27$ & 60 & $6,6-15$ & $50-3000$ \\
\hline $\mathrm{Zn}$ & $50-1450$ & $1-42$ & $1,3-25$ & $15-250$ & $700-49000$ \\
\hline $\mathrm{Fe}$ & - & - & $275-10200$ & - & - \\
\hline Referencia & \multicolumn{2}{|c|}{$\begin{array}{c}\text { Cheraghi et al., (2012) } \\
\text { Nacke, et al., (2013) }\end{array}$} & $\begin{array}{l}\text { Vidal et al., } \\
\text { (2004) }\end{array}$ & $\begin{array}{c}\text { Singh y } \\
\text { Kalamdhad, } \\
(2013)\end{array}$ & $\begin{array}{l}\text { Tervahauta } \\
\text { et al., (2014) }\end{array}$ \\
\hline
\end{tabular}


Tabla 2. Metales pesados en sustratos agrícolas evaluados en diferentes regiones de Colombia.

\begin{tabular}{|c|c|c|c|c|c|c|c|c|c|c|c|c|c|c|}
\hline Matriz de Estudio & $\mathrm{Cd}$ & $\mathrm{Cr}$ & $\mathrm{Hg}$ & $\mathrm{Ni}$ & $\mathrm{Pb}$ & Zn & Mn & Co & As & $\mathrm{Fe}$ & $\mathrm{Na}$ & $\mathbf{M g}$ & $\mathrm{Cu}$ & Referencia \\
\hline Suelo Agrícola & $x$ & $x$ & $x$ & & & & & & & & & & & Bonilla et al., 1991 \\
\hline $\begin{array}{l}\text { Suelo agrícola - } \\
\text { Tejido vegetal }\end{array}$ & & & & & & $x$ & $x$ & & & & & & & Casierra \& Poveda, 2005 \\
\hline Suelo agrícola & $x$ & & & & & & & & & & & & & Insuasty et al., 2006 \\
\hline Suelo Agrícola & $x$ & & & & & & & & & & & & & Insuasty et al., 2008 \\
\hline Suelo Agrícola & $X$ & & & & & & & & $X$ & & & & & Barragán, 2008 \\
\hline Suelo & & $X$ & & $X$ & & $x$ & & & & & & & $x$ & Sánchez et al., 2008 \\
\hline Suelo & & $x$ & & & $x$ & & & & & & & & & Camacho \& Robles, 2009 \\
\hline Suelo & $x$ & $x$ & & & & & & & & & & & & Lora \& Bonilla, 2010 \\
\hline Abonos Orgánicos & $x$ & $x$ & $x$ & $x$ & $x$ & & & & & & & & & Pérez et al., 2010 \\
\hline Suelo Agrícola & $x$ & & $x$ & & & & & & & & & & & Arroyave et al., 2010 \\
\hline Suelo Agrícola & $x$ & & & & $\mathrm{X}$ & & & & & & & & & Martínez \& Palacio, 2010 \\
\hline Lodos & & $X$ & $X$ & & $X$ & & & & & & & & & Soto et al., 2010 \\
\hline $\begin{array}{l}\text { Suelo Agrícola - } \\
\text { Tejido Vegetal }\end{array}$ & $X$ & & $x$ & & $X$ & & & & $X$ & & & & & Miranda et al., 2011 \\
\hline Tejido Vegetal & $x$ & $x$ & & & $\mathrm{X}$ & & & & $X$ & & & & & Bello et al., 2011 \\
\hline $\begin{array}{l}\text { Suelo - Tejido } \\
\text { Vegetal }\end{array}$ & $x$ & $x$ & $x$ & $x$ & & & & & & & & & & Aguirre et al., 2011 \\
\hline Suelo Agrícola & $x$ & & & & & & & & & & & & & Ruíz, 2011 \\
\hline $\begin{array}{l}\text { Suelo, Agua, } \\
\text { Sedimentos }\end{array}$ & $x$ & & & & $X$ & & & & & & & & & $\begin{array}{l}\text { Acosta De Armas \& } \\
\text { Montilla, } 2011\end{array}$ \\
\hline Suelo Agrícola & $x$ & & $x$ & & & & & & & & & & & Arenas \& Hernández, 2012 \\
\hline Suelo & & & & & $x$ & & & & & & & & & Reyes \& Avendaño, 2012 \\
\hline Suelo & & & $x$ & & & & & & & & & & & Román, 2014 \\
\hline Suelo & & & & & & & & & & & & & & Alonso et al.,2014 \\
\hline Suelo & $x$ & & & & & & & & & & & & & Avendaño \& Reyes, 2014 \\
\hline Suelo & $X$ & $x$ & $x$ & & $X$ & & & & $X$ & & & & & Yacomelo, 2014 \\
\hline $\begin{array}{l}\text { Leche-Suelo-Agua- } \\
\text { Tejido Vegetal }\end{array}$ & $x$ & $x$ & $x$ & & $X$ & & & & $x$ & & & & & Londoño, 2014 \\
\hline Suelo Agrícola & $x$ & & & & & & & & & & & & & Bravo et al., 2014 \\
\hline Suelo Agrícola & $x$ & & $x$ & $x$ & $x$ & $x$ & & & & & & & $x$ & Roqueme et al., 2014 \\
\hline Suelo & & & & & $x$ & $x$ & & & & & & & $x$ & Sánchez-Arredondo, 2014 \\
\hline Suelo & $x$ & & & & $x$ & & & & & & & & & Cordero, 2015 \\
\hline Suelo Agrícola & $x$ & $x$ & & $x$ & $X$ & $x$ & & & & & & & $x$ & Mahecha-Pulido et al., 2015 \\
\hline Suelo Agrícola & $x$ & $x$ & & $x$ & $x$ & $x$ & & & & & & & & Ramírez \& Navarro, 2015 \\
\hline Soluciones Acuosas & & $x$ & & & & & & & & & & & & Albis et al., 2015 \\
\hline Tejido Vegetal & & & $x$ & & & & & & & & & & & Argumedo et al., 2015 \\
\hline Suelo Agrícola & & & & & & $x$ & $x$ & & & $\mathrm{X}$ & & & $x$ & Jamioy-Orozco et al., 2015 \\
\hline
\end{tabular}




\begin{tabular}{|c|c|c|c|c|c|c|c|c|c|c|c|c|c|c|}
\hline Matriz de Estudio & Cd & $\mathrm{Cr}$ & $\mathrm{Hg}$ & $\mathrm{Ni}$ & $\mathrm{Pb}$ & $\mathrm{Zn}$ & Mn & Co & As & $\mathrm{Fe}$ & $\mathrm{Na}$ & $M g$ & $\mathrm{Cu}$ & Referencia \\
\hline Tejido Vegetal & & $x$ & $x$ & & & & & & & & & & $x$ & Vergara \& Rodríguez, 2015 \\
\hline Tejido Vegetal & & & & & $x$ & & & & & & & & & Pérez et al., 2015 \\
\hline Tejido Vegetal & & & & & $x$ & & & & & & & & & Vega \& Salamanca, 2016 \\
\hline Tejido Vegetal & $x$ & & & & $x$ & & & & & & & & & Peláez- Peláez et al., 2016 \\
\hline Tejido Vegetal & & & & & & & & & & $x$ & $x$ & $x$ & $x$ & Castillo et al., 2016 \\
\hline Suelo & $x$ & & & $x$ & $x$ & $x$ & & & & & & & $x$ & Cortes et al., 2016 \\
\hline $\begin{array}{l}\text { Tejido vegetal - } \\
\text { seres humanos }\end{array}$ & $x$ & $x$ & $x$ & & $x$ & & & & $x$ & & & & & Estupiñan, 2016 \\
\hline Suelo Agrícola & $x$ & & & $x$ & $x$ & $x$ & & & & & & & $x$ & Cortes et al., 2017 \\
\hline Tejido Vegetal & & & $x$ & & & & & & & & & & & Díaz-Fernández et al., 2017 \\
\hline Suelo Agrícola & & & & & & & & & & $x$ & & & $x$ & Villa et al., 2017 \\
\hline Suelo Agrícola & & & $x$ & & & & & & & $x$ & & & $x$ & Martínez et al., 2017 \\
\hline Suelo Agrícola & $x$ & & & $x$ & $x$ & $x$ & & & & & & & $x$ & Trujillo-González et al., 2017 \\
\hline Suelo Agrícola & $x$ & & & $x$ & $x$ & $x$ & & & & & & & $x$ & Marrugo-Negrete et al., 2017 \\
\hline Suelo Agrícola & $x$ & & & & & & & & & & & & & Rodríguez, 2017 \\
\hline Suelo & & & $x$ & & & & & & & & & & & Díaz et al., 2017 \\
\hline
\end{tabular}

Fuente: los autores

En la mayoría de estos estudios se reitera la dificultad para concluir si las áreas estudiadas pueden catalogarse como "áreas contaminadas" debido a la ausencia de valores que puedan usarse como referencia (Rueda et al., 2011; Mahecha-Pulido et al., 2015). Los resultados encontrados están en consonancia con lo planteado por Alloway, donde propone que los metales pesados de mayor interés por estar asociados a la polución ambiental, los daños en la agricultura y la salud humana son, el arsénico (As), el cadmio (Cd), el mercurio $(\mathrm{Hg})$, el plomo $(\mathrm{Pb})$, el talio $(\mathrm{Tl})$ y el uranio $(U)$. En los estudios realizados en Colombia se analizan los siguientes metales: cadmio (Cd), es uno de los elementos con alto potencial de contaminación; el mercurio $(\mathrm{Hg})$ el cual a escala global tiene como principales fuentes actividades antropogénicas, el uso de combustibles fósiles y la explotación de oro (World Health Organization, 2007), en Colombia constituye un riesgo ambiental y de salud pública, principalmente en extracciones ilegales (Olivero y Jhonson 2002); por su lado, el plomo $(\mathrm{Pb})$, es uno de los metales más tóxicos y comunes en el planeta, debido a que es utilizado en productos tan comunes como baterías, pinturas, tuberías e insecticidas, y es residuo de actividades como el uso de combustibles fósiles, la incineración de basura y la producción metalúrgica (Sánchez, 2010). Finalmente el cromo $(\mathrm{Cr})$ aunque no está considerado por Alloway (1995), como de importancia para estudios ambientales, debido a que es un elemento esencial, este presenta un riesgo, especialmente en zonas con importante dinámica industrial como las cuencas de los ríos Medellín y Bogotá (Sánchez, 2010).

En la figura 1, se observa que la mayor parte de los estudios se concentran en la región Andina y región Caribe con una carencia de éstos en la zona oriental (región de la Orinoquia). Según esto, los departamentos donde se concentran los estudios en orden por cantidad son: Cundinamarca $>$ Córdoba $=$ Antioquia $>$ Valle del Cauca $=$ Meta $>$ Boyacá $=$ Santander $>$ Sucre $=$ Atlántico $=$ Bolívar $=$ Cauca $>$ Nariño. En la sección $b$, se ubican los estudios en los que se analizaron el plomo, principalmente en el departamento de Cundinamarca, debido a que en los sistemas agrícolas se capta para el riego aguas contaminadas resultantes de procesos industriales (Soto et al., 2010; Miranda, 2011) y también, por el uso excesivo de insumos agrícolas y maquinaria (Yacomelo, 2014; Roqueme et al., 2014; Mahecha-Pulido et al., 2015). En la sección c, están ubicados los estudios en los que se analizó el mercurio, donde se establece la influencia que tiene la minería sobre la acumulación de este metal pesado en los sistemas agrícolas (Román, 2014; Roqueme et al., 2014; Argumedo et al., 2015; Martinez et al., 2017), en los departamentos de Antioquia, Córdoba, Sucre, Bolívar y Atlántico, zonas del país que tienen una alta afectación por la explotación minera ilegal (PinedoHernández et al., 2015; García et al., 2015; Marrugo- 


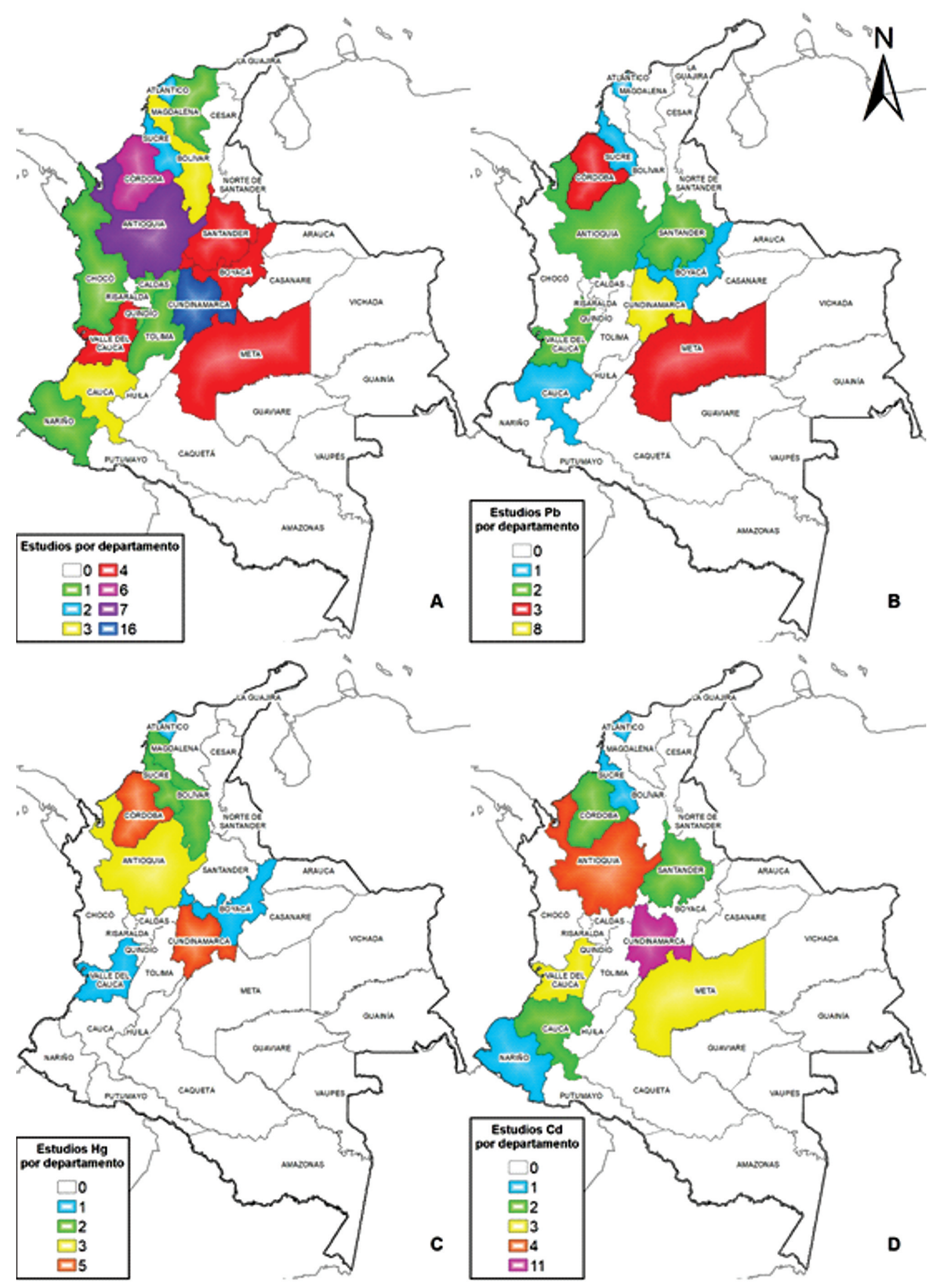

Figura 1. Distribución de estudios en Colombia. A) estudios por departamento. B) estudios de plomo Pb. C) estudios de mercurio Hg. D) estudios de cadmio Cd. 
Negrete et al., 2017), mientras que en el departamento de Cundinamarca los estudios se relacionan al uso de aguas provenientes de afluentes contaminados (Soto et al., 2010). En la sección d, se encuentran los estudios relacionados con el cadmio, elemento que se analiza en el $88 \%$ de los estudios recopilados, una las principales fuentes es el uso persistente de fertilizantes fosforados (Mahecha-Pulido et al., 2015; Ramírez y Navarro, 2015; Rodríguez, 2017), además del asocio de este elemento con aguas contaminadas usadas para riego, en el caso del departamento de Cundinamarca (Ruíz, 2011; Bello et al., 2011; Acosta De Armas y Montilla, 2011).

\section{Perspectivas y desafios}

La contaminación generada por las actividades del ser humano como los desechos que se producen en los asentamientos y las industrias, las emisiones de los automóviles y los productos utilizados en su funcionamiento, la actividad minera, y la aplicación de fertilizantes y pesticidas agrícolas, están contribuyendo a una continua acumulación de metales pesados en los suelos (Tu et al., 2000; Nouri et al., 2008). El crecimiento de la producción agrícola mundial se estima que será de un 1,5 por ciento al año de promedio durante la próxima década, donde la oferta de productos básicos agrícolas deberá crecer al ritmo de la demanda mundial (FAO, 2015). Por esta razón, aumentarán las aplicaciones de fertilizantes y pesticidas, fuente importante de metales pesados en las zonas agrícolas del mundo, (Alloway, 2013).

Para el caso colombiano, el gobierno tiene el reto de preservar y reorganizar el uso del suelo específicamente en las zonas agrícolas, que según datos de la Unidad de Planificación rural Agropecuaria (2014), se tienen 22 millones de hectáreas con potencial agrícola de los cuales apenas se están usando 5,3 millones de hectáreas, que refleja el problema de ineficiencia del uso del suelo rural. Al explotar el potencial agrícola que tiene Colombia, será necesario incrementar la aplicación de insumos y el uso maquinaria que podrá tener como efecto el aumento del riesgo de contaminación. En este sentido la información científica sobre la base geoquímica de los metales pesados será determinante para establecer programas de monitoreo y seguimiento. Asimismo, Rueda et al., (2011) y Mahecha-Pulido et al., (2015), plantean que la principal problemática en el país es la inexistencia de una regulación normativa de los metales pesados en los zonas agrícolas, por lo que sería indispensable la generación de estándares en los que se establezcan los índices de contaminación con va- lores normales y valores excesivos, modelados para los suelos, la determinación de su aptitud para los diferentes usos y los riesgos que se pueden presentar en los ecosistemas circundantes.

En el mundo, la generación de valores de referencia de metales pesados se ha convertido en un instrumento para la gestión de los suelos, ya que con esto se distingue entre las condiciones naturales y aportes derivados de diferentes actividades antrópicas. En Colombia no se cuenta con valores propios por lo cual todos los investigadores han comparados sus resultados con valores de referencia propuestos por organizaciones como la Agencia de Protección Ambiental EPA (1996), la Organización Mundial para la Salud OMS y la Organización para la Agricultura y la Alimentación FAO (OMS-FAO, 1992), o por países específicos como Holanda (Brus et al., 2009), Brasil (Fadigas et al., 2006), España (Mico et al., 2005), entre otros.

\section{Conclusiones}

Establecer los valores de referencia para Colombia, es importante como primer paso para enfrentar el riesgo de salud pública que representan los metales pesados, y se debe generar estudios que permitan establecer los valores de referencia de calidad y de contaminantes, que aporten datos que puedan ser integrados en el proceso de gestión del recurso suelo.

Es necesario que en Colombia se cree una norma técni$\mathrm{ca}$, indispensables para evaluar la calidad de los suelos, que tenga en cuenta la implementación de valores de referencia para parámetros químicos, físicos y biológicos propios. Es una tarea complicada, pero esencial, ya que, la generación de conocimiento es uno de los pasos esenciales para los procesos de gobernabilidad, en este caso del recurso suelo en el Colombia.

Es necesario que para regiones como la Orinoquia, denominada como frontera agrícola del país, se promuevan los estudios referentes a los posibles impactos que se pueden ocasionar debido a la intensificación de la producción, en la salud de los suelos, los sistemas naturales y sus habitantes.

La conservación del suelo debe convertirse en un tema prioritario para el país debido a que este recurso oferta servicios ecosistémicos que suplen necesidades ambientales, económicas y sociales para al bienestar no solo de sociedad colombiana sino mundial.

\section{Bibliografía}

Acosta De Armas MM, Montilla Peña JX. 2011. Evaluación de la contaminación por cadmio y plomo en agua, suelo, y sedimen- 
to y análisis de impactos ambientales en la subcuenca del rio Balsillas afluente del rio Bogotá. (Tesis Pregrado), Facultad de Ingeniería, Universidad de la Salle. Bogotá: Universidad Libre. Recuperado de http://repository. lasalle. edu. co/bitstream/ handle/10185/14892, 41

Adriano DC. 2001. Trace Elements in Terrestrial Environments: Biogeochemistry, Bioavailability and Risks of Metals. 2nd Edition, Springer, New York. Pp. 867. http://dx.doi.org/10.1007/978-0387-21510-5

Aguirre W, Fischer G, Miranda D. Tolerancia a metales pesados a través del uso de micorrizas arbusculares en plantas cultivadas. Rev Colomb Cienc Hortic. 2011;5(1):141-153.

Albis A, Cajar L, Domínguez M. Análisis cinético de la adsorción de $\mathrm{Cr}(\mathrm{VI})$ en soluciones acuosas a concentraciones de 10-20 $\mathrm{mg} / \mathrm{L}$ con el uso de cáscara de yuca amarga (Manihot esculenta). Prospectiva. 2015;13(2):64-71.

Alloway BJ. 1995. Heavy Metals in Soils, second ed. Blackie Academic \& Professional, London. Pp. 368

Alloway BJ. Heavy Metals in Soils - Trace Metals and Metalloids in Soils and their Bioavailability (3ra Ed.) United Kingdom. Environ Pollut. 2013;22:11-50.

Alonso D, Latorre S, Castillo E, Brandão P. Environmental occurrence of arsenic in Colombia: A review. Environ Pollut. 2014;186:272-281.

Arenas S, Hernández A. 2012. Fitotoxicidad del Cadmio (Cd) y el Mercurio (Hg) en la especie Brassica nigra (Tesis Pregado), Universidad de Medellín.

Argumedo MP, Vergara C, Vidal J, Marrugo JL. Evaluación de la concentración de mercurio en arroz (Oryza sativa) crudo y cocido procedente del municipio de San Marcos- Sucre y zona aurífera del municipio de Ayapel - Córdoba. Rev Univ Ind Santander Salud. 2015;47(2):169-177.

Arroyave C, Araque P, Peláez C. Evaluación de la bioacumulación y toxicidad de cadmio y mercurio en pasto llanero (Brachiaria dictyoneura). Vitae. 2010;17(1):45-49.

Avendaño G, Reyes M. 2014. Gestión del proceso de técnica diagnóstica sobre el riesgo que representa el cadmio en el suelo. LACCEI (Latin American and Caribbean Consortium of Engineering Institutions). Recuperado de sitio web: http://www.laccei. org/LACCEI2014-Guayaquil/RefereedPapers/RP056.pdf

Barragán O. Estudio de diferentes metodologías para determinar la biodisponibilidad de cadmio y arsénico en suelos y su relación con la concentración en plantas. NOVA. 2008;6(9):35-39.

Bello P, Lesmes L, Fischer G. Determinación de metales pesados en apio (Apium graveolens L.), lechuga (Lactuca sativa L. var. Batavia) y acelga (Beta vulgaris L.) mediante icp-aes en dos zonas de producción de hortalizas de la Sabana de Bogotá. $2011 ; 1: 114$

Bonilla CRC, García Á, Castillo LE. Adsorción de cadmio, cromo y mercurio en suelos del Valle del Cauca a varios valores de $\mathrm{pH}$. Acta Agron. 1991;41(1-4): 60-78.

Bouma. 2012. Hydropedology as a powerful tool for environmental policy and regulations; towards sustainable land use, manage- ment and planning. En: Lin, H. (Ed.), Hydropedology: Synergistic Integration of Soil Science and Hydrology. Academic Press, Elsevier B.V., pp. 483-512. http://dx.doi.10.1016/B978-0-12386941-8.00015-0

Bravo I, Arboleda C, Martín F. Efecto de la calidad de la materia orgánica asociada con el uso y manejo de suelos en la retención de cadmio, en sistemas altoandinos de Colombia. Acta Agron. 2014;63(2):164-174.

Brevik EC, Cerdà A, Mataix-Solera J, Pereg L, Quinton JN, Six J, Van Oost K. The interdisciplinary nature of SOIL. Soil. 2015;1(1):117.

Brus D, Lame F, Nieuwenhuis R. National baseline survey of soil quality in the Netherlands. Environ Pollut. 2009;157(7):20432052.

Buol SW, Southard RJ, Graham RC, McDaniel PA. 2011. Soil genesis and classification. John Wiley \& Sons. Pp.543

Camacho - Angel JP, Robles - Cruz LF. 2009. Diagnóstico ambiental de la contaminación del suelo en el municipio de Chocontá y prueba piloto con dos de los contaminantes más representativos bioacumulados en arveja, haba y pasto ray grass. (Tesis de pregrado), Facultad de Ingeniería, Universidad de la Salle.

Casierra F, Poveda J. La toxicidad por exceso de Mn y Zn disminuye la producción de materia seca, los pigmentos foliares y la calidad del fruto en fresa (Fragaria sp. cv. Camarosa). Agron Colomb. 2005;23(2):285-289.

Castillo JE, Moreno DM, Ramírez MV. Evaluación del contenido de los metales $\mathrm{Cu}, \mathrm{Mg}$, Fe y Na, presentes en el zapote (Quararibea Cordata), provenientes del Valle del Cauca, Colombia. Ingeniería solidaria. 2016;12(19):37-48.

Ceccon E. tragedia en dos actos La revolución verde. Ciencias. 2008;1(91):21-29.

Cheraghi M, Lorestani B, Merrikhpour H. Investigation of the effects of phosphate fertilizer application on the heavy metal content in agricultural soils with different cultivation patterns. Biol Trace Elem Res. 2012;145(1):87-92.

COM. 2002. Comunicación de la Comisión al Consejo, el Parlamento Europeo, el Comité Económico y Social y el Comité de las Regiones. Hacia una estrategia temática para la protección del suelo. COM (2002) 179 final.

Cordero J. 2015. Fitorremediación in situ para la recuperación de suelos contaminados por metales pesados (plomo y cadmio) y evaluación de selenio en la finca Furatena alta en el municipio de Útica (Cundinamarca). (Tesis Pregrado), Facultad de Ingeniería, Universidad libre de Colombia.

Cortés LE, Martin FJ, Sarria MM. Evaluación de la toxicidad de metales pesados en dos suelos agrícolas de Colombia mediante bioensayos. Temas Agrarios. 2017; 22(2):42-45.

Cortes L, Bravo I, Martin F, Menjivar J. Extracción secuencial de metales pesados en dos suelos contaminados (Andisol y Vertisol) enmendados con ácidos húmicos. Acta Agronómica. 2016;65(3):232-238. 
Departamento Nacional de Planeación República de Colombia (DNP). 2014. Consejo Nacional de Política Económica y Social (CONPES 3797). Política para el desarrollo integral de la Orinoquia: Altillanura- fase I.

Díaz L, Vidal J, Marrugo J. 2017. Evaluación de la capacidad acumuladora de la leguminosa Gliricidia sepium en suelos contaminados por mercurio (Hg). Memorias III Seminario Internacional de Ciencias Ambientales SUE-Caribe. Pp. 184-187.

Díaz-Fernandez L, Camelo-Furnieles C, Durango-Hernández J, Urango-Cardenas I, Marrugo-Negrete J. 2017. Evaluación de plantas nativas en la región de La Mojana como fitorremediadoras de suelos impactados por mercurio. In. Memorias III Seminario Internacional de Ciencias Ambientales SUE-Caribe. Pp. 84-86.

Doran JW, Parkin TB. 1994. Defining and assessing soil quality. In: Doran, J.W., Coleman, D.C., Bezdicek, D.F., Stewart, B.A. (Eds.), Defining Soil Quality for a Sustainable Environment. SSSA Special Publication No. 35, ASA and SSSA, Madison, WI. Pp. 3-21.

Duval ME, Galantini JA, Iglesias JO, Canelo S, Martinez JM, Wall L. Analysis of organic fractions as indicators of soil quality under natural and cultivated systems. Soil Tillage Res. 2013;131:11-19.

Estupiñan J. 2016. Evaluación del riesgo en la salud humana por consumo de vegetales irrigados con aguas que contienen metales pesados en un sector de la cuenca del río Tunjuelo (Tesis Maestría), Universidad Nacional de Colombia-Sede Bogotá.

Fadigas FDS, Amaral Sobrinho ND, Mazur N, Anjos LD, Freixo AA. Proposição de valores de referência para a concentração natural de metais pesados em solos brasileiros. Rev Bras Eng Agr Amb. 2006;10(3): 699-705.

FAO O. 1992. Evaluación de diversos aditivos alimentarios y los contaminantes mercurio, plomo y cadmio. Información Técnica, 50.

Garbisu C, Becerril JM, Epelde L, Alkorta L. Bioindicadores de la calidad del suelo: herramienta metodológica para la evaluación de la eficacia de un proceso fitorremediador. Ecosistemas. 2007; 16(2):44-49.

García O, Veiga MM, Cordy P, Suescún OE, Molina JM, Roeser M. Artisanal gold mining in Antioquia, Colombia: a successful case of mercury reduction. J Clean Prod. 2015;90:244-252.

Gutiérrez C, Fernández C, Escuer M, Campos-Herrera R, Rodríguez MEB, Carbonell G, Martín JAR. Effect of soil properties, heavy metals and emerging contaminants in the soil nematodes diversity. Environ Pollut. 2016;213: 184-194.

Hartemink AE, McBratney A. A soil science renaissance. Geoderma. 2008;148(2):123-129.

Insuasty L, Burbano $\mathrm{H}$, Menjivar J. Movilidad del cadmio en suelos cultivados con trigo en Tangua, Nariño, Colombia. Acta agronómica. 2006;55(2):29-32.

Insuasty L, Burbano $\mathrm{H}$, Menjivar J. Dinámica del cadmio en suelos cultivados con papa en Nariño, Colombia. Acta Agronómica. 2008;57(1):51-54.

Jamioy D, Menjivar - Flores J, Rubiano Y. Indicadores químicos de calidad de suelos en sistemas productivos del Piedemonte de los Llanos Orientales de Colombia. Acta Agronómica. 2015;64(4):302-307.
Jenny H. 1994. Factors of soil formation: a system of quantitative pedology. Courier Corporation. Foreword by Ronald Amundson, University of California, Berkeley. Dover Publications, INC. New York

Kabata-Pendias, A. Soil-plant transfer of trace elements-an environmental issue. Geoderma. 2004;122(2):143-149.

Karlen DL, Mausbach MJ, Doran JW, Cline RG, Harris RF, Schuman GE. Soil quality: a concept, definition and framework for evaluation. Soil Sci Soc Am J. 1997;61:4-10.

Khaledian Y, Pereira P, Brevik EC, Pundyte N, Paliulis D. The influence of organic carbon and $\mathrm{pH}$ on heavy metals, potassium, and magnesium levels in Lithuanian Podzols. Land Degrad Dev. 2017;28(1):345-354.

Londoño L. 2014. Presencia de metales pesados en hatos lecheros de los municipios de San Pedro y Entrerríos, Antioquia, Colombia (Tesis Doctoral), Universidad León, España.

Lora Silva R, Bonilla - Gutiérrez H. Remediación de un suelo de la cuenca alta del río Bogotá contaminado con los metales pesados cadmio y cromo. Revista UDCA Actualidad \& Divulgación Científica. 2010;13(2):61-70.

Mahecha-Pulido JD, Trujillo-González JM, Torres-Mora MA. Contenido de metales pesados en suelos agrícolas de la región del Ariari, Departamento del Meta. Orinoquia. 2015;19(1):118-122.

Marrugo-Negrete J, Pinedo-Hernández J, Díez S. Assessment of heavy metal pollution, spatial distribution and origin in agricultural soils along the Sinú River Basin, Colombia. Environ. Res. 2017;154:380-388.

Martínez G, Palacio C. 2010. Determinación de metales pesados $\mathrm{Cd}$ y $\mathrm{Pb}$ en suelos y granos de cacao frescos y fermentados mediante espectroscopia de absorción atómica de llama. (Tesis Pregrado), universidad Industrial de Santander, Colombia.

Martínez Z, González M. Contaminación de suelos agrícolas por metales pesados, zona minera El Alacrán, Colombia. Temas Agrarios. 2017;22(2):21-31.

McBratney A, Field DJ, Koch A. The dimensions of soil security. Geoderma. 2014;213:203-213.

Mico C. 2005. Estudio de Metales Pesados en Suelos Agrícolas con Cultivos Hortícolas de la Provincia de Alicante. (Tesis Doctoral). Universidad de Valencia. Valencia - España.

Mico C, Peris M, Recatalá L, Sánchez J. Baseline values for heavy metals in agricultural soils in an European Mediterranean region. Sci Total Environ. 2007;378(1):13-17.

MINISTERIO DE AGRICULTURA Y DESARROLLO RURAL. 2014. Unidad de Planificación Rural Agropecuaria. Presentación Institucional. República de Colombia. Recuperado en https://www.minagricultura.gov.co/Documents/UPRA_Oferta_Institucional.pdf

Miranda D, Carranza C, Rojas CA, Jerez CM, Fischer G, Zurita J. Acumulación de metales pesados en suelo y plantas de cuatro cultivos hortícolas, regados con agua del río Bogotá. Rev Colomb Cienc Hortic. 2011;2(2):180-191.

Montanarella L, Jones RJA. 1999. The European Soil Bureau, in P. Bullock, R.J.A. Jones, and L. Montanarella (eds.), Soil Resour- 
ces of Europe, European Soil Bureau Research Report No.6, EUR 18991 EN, Office for Official Publications of the European Communities, Luxembourg, p. 3-14.

Nacke H, Gonçalves Jr AC, Schwantes D, Nava IA, Strey L, Coelho GF. Availability of heavy metals $(\mathrm{Cd}, \mathrm{Pb}$, and $\mathrm{Cr})$ in agriculture from commercial fertilizers. Arch Environ Con Tox. 2013;64(4):537-544.

Nava-Ruíz C, Méndez-Armenta M. Efectos neurotóxicos de metales pesados (cadmio, plomo, arsénico y talio). Arch Neurocien (Mex). 2011;16(3):140-147.

Nouri J, Mahvi AH, Jahed GR, Babaei A. A regional distribution pattern of groundwater heavy metals resulting from agricultural activities. Environmental Geology, 55, 1337-1343.lomo, arsénico y talio). Arch Neurocien (Mex). 2008;16(3):140-147.

Olivero J, Johnson B, Arguello E. Human exposure to mercury in San Jorge river basin, Colombia (South America). Sci Total Environ. 2002;289(1):41-47.

Organización de las Naciones Unidas para la Alimentación y la Agricultura (FAO). 2015. El suelo es un recurso no renovable su conservación es esencial para la seguridad alimentaria y nuestro futuro sostenible

Peláez-Peláez M, Bustamanete J, Gómez E. Presencia de cadmio y plomo en suelos y su bioacumulación en tejidos vegetales en especies de brachiaria en el Magdalena medio colombiano. Revista Luna Azul. 2016;(43):82-101.

Pérez A, Barraza Z, Martínez D. Identificación de bacterias endófitas resistentes a plomo, aisladas de plantas de arroz. Agron Mesoam. 2015;26(2):267-276.

Pérez R, Pérez A, Vertel M. Caracterización nutricional, fisicoquímica y microbiológica de tres abonos orgánicos para uso en agroecosistemas de pasturas en la subregión sabanas del departamento de Sucre, Colombia. Tumbaga. 2010. 1(5):2737.

Pinedo-Hernández J, Marrugo-Negrete J, Díez S. Speciation and bioavailability of mercury in sediments impacted by gold mining in Colombia. Chemosphere. 2015; 119:1289-1295.

Ramírez MN, Navarro MR. Análisis de metales pesados en suelos irrigados con agua del río Guatiquía. Ciencia en Desarrollo. 2015;6(2):167-175.

Reyes M, Avendaño G. Estudio ambiental sobre el riesgo ecológico que representa el plomo presente en el suelo. Revista EAN. 2012;(72):66-75.

Rodríguez Albarracín HS. 2017. Dinámica del cadmio en suelos con niveles altos del elemento, en zonas productoras de cacao de Nilo y Yacopí, Cundinamarca (Doctoral dissertation), Universidad Nacional de Colombia-Sede Bogotá.

Román L. 2014. Determinación de los niveles de mercurio en suelo en San Martín de Loba, sur de Bolívar-Colombia. Memorias del II Seminario de Ciencias Ambientales Sue-Caribe \& VII Seminario Internacional de Gestión Ambiental. Pp.143-149. ISBN: 978-958-9244-64-7

Roqueme J, Pinedo J, Marrugo J, Aparicio A. 2014. Metales pesados en suelos agrícolas del valle medio y bajo del rio Sinú, departa- mento de Córdoba. Memorias del II Seminario de Ciencias Ambientales Sue-Caribe \& VII Seminario Internacional de Gestión Ambiental. Pp.106-107. ISBN: 978-958-9244-64-7

Rueda GS, Rodríguez JAV, Madriñán RM. Metodologías para establecer valores de referencia de metales pesados en suelos agrícolas: Perspectivas para Colombia. Acta Agronómica. 2011;60(3):203-217.

Ruíz J. Evaluación de tratamientos para disminuir cadmio en lechuga (Lactuca sativa L.) regada con agua del río Bogota. Rev Colomb Cienc Hortic. 2011;5(2):233-243.

Sam K, Coulon F, Prpich G. Working towards an integrated land contamination management framework for Nigeria. Sci Total Environ. 2016;571:916-925.

Sánchez MS. 2010. Contaminación por Metales Pesados en el Botadero de Basuras de Moravia en Medellín, Transferencia a Flora y Fauna y Evaluación del Potencial Fitoregulador de Especies Nativas e Introducidas. (Tesis Doctoral). Pontificia Universidad Javeriana. Bogotá - Colombia.

Sánchez LH, González LM, Espinosa A. Modelación de elementos traza en el horizonte a de suelos, plancha 170 (vélez, departamentos de Santander y Boyacá), DYNA. 2008;75(156):157164.

Sánchez-Arredondo LH. Distribución de los elementos Cu-Pb$\mathrm{Zn}$ en suelos de la plancha 168 (Argelia). Bol Cienc Tierra. 2014;(36):10-17.

Singh J, Kalamdhad AS. Assessment of bioavailability and leachability of heavy metals during rotary drum composting of green waste (Water hyacinth). Ecol Eng. 2013;52:59-69.

Soto C, Gutiérrez S, Rey-León A, González-Rojas E. Biotransformación de metales pesados presentes en lodos ribereños de los ríos Bogotá y Tunjuelo. NOVA. 2010;8(14):195-205.

Tervahauta T, Rani S, Leal LH, Buisman CJ, Zeeman G. Black water sludge reuse in agriculture: Are heavy metals a problem?. J Hazard Mater. 2014; 274:229-236.

Tiller KG. Heavy metals in soils and their environmental significance. Adv Soil Sci. 1989;9:113-142.

Trujillo-González JM, Mahecha-Pulido JD, Torres-Mora MA, Brevik EC, Keesstra SD, Jiménez-Ballesta R. Impact of potentially contaminated river water on agricultural irrigated soils in an equatorial climate. Agriculture. 2017;7(7):52.

Trujillo-González JM, Torres-Mora MA, Keesstra S, Brevik EC, Jiménez-Ballesta R. Heavy metal accumulation related to population density in road dust samples taken from urban sites under different land uses. Science of the Total Environment, 2016;553:636-642.

Tu C, Zheng CR, Chen H M. Effectofapplying chemical fertilizers on forms of lead and cadmium in red soil. Chemosphere. 2000:41:133-138.

US EPA. 1996. Ecotox thresholds. ECO Update, Intermittent Bulletin 3(2). EPA 540/F-96-038. US Environmental Protection Agency, Office of Emergency and Remedial Response, Washington, DC 
Vega D, Salamanca Á. Contenidos de plomo en acelga común beta vulgaris I., producida en el contexto de la agricultura urbana (Bogotá, Colombia). Rev Luna Azúl. 2016;42:44-53.

Vergara E, Rodríguez P. Presencia de mercurio, plomo y cobre en tejidos de Orechromis niloticus: sector de la cuenca alta del Río Chicamocha, vereda Volcán, Paipa, Colombia. Producctión + Limpia. 2015:10(2):114-126.

Vidal J, Pérez-Sirvent C, Martínez-Sánchez MJ, Navarro MC. Origin and behaviour of heavy metals in agricultural Calcaric Fluvisols in semiarid conditions. Geoderma. 2004;121(3):257-270.

Villa M, Martínez E, Cartagena J, Rodríguez O, Osorio N. Characterization of soils cultivated with rubber in the Colombian
Bajo Cauca Antioqueño region. Rev Fac Nac Agron Medellin. 2017;70(2):8155-8167.

World Health Organization, Regional Office for Europe, 2007. Health risks of heavy metals from long-range transboundary air pollution. Copenhagen, Denmark.

Yacomelo M. 2014. Riesgo toxicológico en personas expuestas, a suelos y vegetales, con posibles concentraciones de metales pesados, en el sur del Atlántico, Colombia. (Tesis Maestría), Universidad Nacional de Colombia.

Yi YM, Sung K. Influence of washing treatment on the qualities of heavy metal-contaminated soil. Ecol Eng. 2015;81:89-92. 